\title{
Clinical Features and Outcomes of Patients with Dementia Compared to an Aging Cohort Hospitalized During the Initial New York City COVID-19 Wave
}

\author{
Amro A. Harb ${ }^{\mathrm{a}}$, RuiJun Chen ${ }^{\mathrm{b}, \mathrm{c}}$, Herbert S. Chase ${ }^{\mathrm{b}}$, Karthik Natarajan ${ }^{\mathrm{b}, \mathrm{d}, \mathrm{e}}$ \\ and James M. Noble ${ }^{\mathrm{d}, \mathrm{e}, \mathrm{f}, \mathrm{g}, *}$ \\ ${ }^{a}$ Columbia University Vagelos College of Physicians and Surgeons, New York, NY, USA \\ ${ }^{\mathrm{b}}$ Department of Biomedical Informatics, Columbia University, New York, NY, USA \\ ${ }^{\mathrm{c}}$ Department of Translational Data Science and Informatics, Geisinger, Danville, PA, USA \\ ${ }^{\mathrm{d}}$ Columbia University Irving Medical Center, New York, NY, USA \\ ${ }^{\mathrm{e}}$ New York-Presbyterian Hospital, New York, NY, USA \\ ${ }^{\mathrm{f}}$ Taub Institute for Research on Alzheimer's Disease and the Aging Brain, GH Sergievsky Center, \\ New York, NY, USA \\ ${ }^{\mathrm{g}}$ Department of Neurology, Vagelos College of Physicians and Surgeons, New York, NY, USA
}

Accepted 4 March 2021

Pre-press 13 March 2021

\begin{abstract}
.
Background: Patients with dementia are vulnerable during the coronavirus disease 2019 (COVID-19) pandemic, yet few studies describe their hospital course and outcomes.

Objective: To describe and compare the hospital course for COVID-19 patients with dementia to an aging cohort without dementia in a large New York City academic medical center.

Methods: This was a single-center retrospective cohort study describing all consecutive patients age 65 or older with confirmed COVID-19 who presented to the emergency department or were hospitalized at New York-Presbyterian/Columbia University Irving Medical Center between March 6 and April 7, 2020.

Results: A total of 531 patients were evaluated, including 116 (21.8\%) with previously diagnosed dementia, and 415 without dementia. Patients with dementia had higher mortality $(50.0 \%$ versus $35.4 \%, p=0.006)$; despite similar comorbidities and complications, multivariate analysis indicated the association was dependent on age, sex, comorbidities, and code status. Patients with dementia more often presented with delirium $(36.2 \%$ versus $11.6 \%, p<0.001)$ but less often presented with multiple other COVID-19 symptoms, and these findings remained after adjusting for age and sex.

Conclusion: Hospitalized COVID-19 patients with dementia had higher mortality, but dementia was not an independent risk factor for death. These patients were approximately 3 times more likely to present with delirium but less often manifested or communicated other common COVID-19 symptoms. For this high-risk population in a worsening pandemic, understanding the unique manifestations and course in dementia and aging populations may help guide earlier diagnosis and optimize medical management.
\end{abstract}

Keywords: Advance directives, COVID-19, delirium, dementia, geriatrics, neurology

\footnotetext{
${ }^{*}$ Correspondence to: James M. Noble, MD, MS, CPH, FAAN, Associate Professor of Neurology at CUIMC, Taub Institute for Research on Alzheimer's Disease and the Aging Brain, G.H. Sergievsky Center, Department of Neurology, Columbia
}

University Irving Medical Center, 710 W. 168th St Box 176, New York, NY 10032, USA. Tel.: +1 212342 4126; E-mail: jn2054@ columbia.edu. 


\section{INTRODUCTION}

The coronavirus disease 2019 (COVID-19) is a global pandemic, and persons with dementia are particularly vulnerable [1]. As of January 9, 2021, the Centers for Disease Control and Prevention (CDC) reported 41,394 excess deaths among persons with Alzheimer's disease and dementia since February 1, 2020 in the United States (US) alone, which far exceeds the excess deaths for any other comorbidities [2]. Despite these increased excess deaths, there have been few studies that focus on understanding how persons with dementia present clinically with COVID-19 or describe their hospital course and outcomes.

Patients with dementia are a unique population who may present differently or be at increased risk of death from COVID-19 [3-11]. Dementia may impact the ability to learn, understand, and retain information regarding the pandemic. This can have important consequences, including increased exposure due to non-adherence to preventative practices; delayed recognition of typical symptoms; less careseeking behavior; and delayed treatment [1, 12]. These patients are typically older, and age has been consistently reported as an independent risk factor for in-hospital death from COVID-19 in many studies [3-5, 13]. Additionally, evidence from a recent multicenter US study and earlier European studies suggests that dementia patients with COVID-19 may be more likely to experience delirium [6, 14-16], an independent risk factor for increased in-hospital mortality $[14,15]$.

Therefore, in hopes of better understanding the illness course of this unique population, we sought to describe the clinical characteristics and hospital course of all adults aged 65 years and older who presented to the emergency department (ED) or were hospitalized with COVID-19 in a quaternary academic medical center in New York City (NYC) during the initial surge of cases in the US COVID-19 pandemic.

\section{METHODS}

\section{Study design and data source}

This study was approved by the Institutional Review Board (IRB) of Columbia University under protocol AAAS9834. We expand on a previously described, retrospective, manually abstracted database of all consecutive patients who presented to the New York-Presbyterian/Columbia University Irving
Medical Center (NYP/CUIMC) ED or were hospitalized at NYP/CUIMC and had a positive result on reverse transcriptase polymerase chain reaction assay for severe acute respiratory syndrome coronavirus-2 (SARS-CoV-2), indicating active COVID-19 illness [17]. Data was manually abstracted from the electronic medical record (EMR) into a Research Electronic Data Capture (REDCap) instrument [17, 18]. In compliance with the IRB-approved protocol, patient consent was not applicable in this study.

The original REDCap database contained information on demographics, body mass index (BMI), comorbidities, and common presenting signs and symptoms as descriptive variables [17]. For outcome variables, the database contained information on status for death, discharge, intubation, level of care, length of stay, and in-hospital complications [17]. Length of stay was defined as the date and time from initial ED presentation to the date and time of either discharge or death.

\section{Data collection and definitions of variables}

Additional abstraction was performed in this study to supplement the previously created REDCap database with descriptive information regarding dementia status, additional signs and symptoms, and code status. Dementia was identified through chart review if Alzheimer's dementia, vascular dementia, Lewy-Body dementia, Parkinson's dementia, or any other dementias of unspecified type were indicated in the EMR provider notes, such as admission notes, progress notes, or discharge summaries. Dementia was not stratified based on severity. Additional signs and symptoms in this study included delirium, chest pain, and abdominal pain. Delirium was identified if the patient was described as altered or confused in the EMR.

Do-Not-Resuscitate/Do-Not-Intubate (DNR/DNI) was indicated by either a) DNR and DNI order or b) DNR order only at death or date of discharge. If no code status was indicated at discharge or death, patients were assumed to be full code. DNR/DNI orders placed before inpatient admission or in prior hospitalizations were also recorded. Patients who were DNR/DNI at discharge or death and were never full code during their hospital course were included in a separate category as "consistent DNR/DNI."

\section{Study sample}

The processed REDCap dataset only included visits with the highest level of care for patients with 


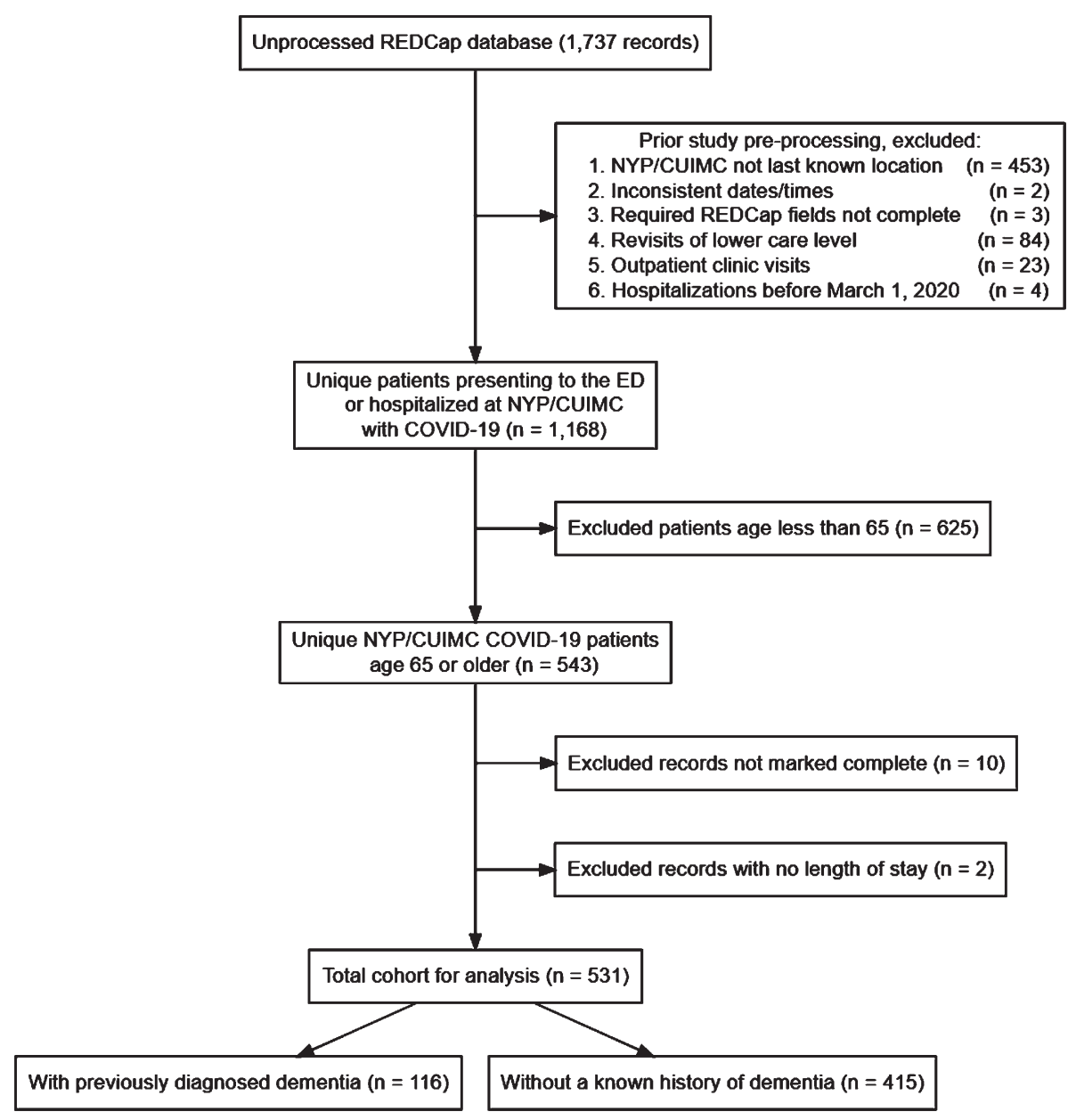

Fig. 1. STROBE Flowchart of Study Sample Selection. Selection of the study sample is presented in a flowchart. The steps from "Unprocessed REDCap database (1,737 records)" to "Unique patients presenting to the ED or hospitalized at NYP/CUIMC with COVID-19 (n=1,168)" were performed with an R script used in the prior study that created the REDCap database [17]. Additional exclusions were made in this study for patients with a presenting age less than 65, with records not marked "Complete" in REDCap, and with missing values for length of stay.

multiple visits [17]. Levels of care in this study included admission to the intensive care unit (ICU), inpatient admission (not ICU), or ED visit only. For patients with multiple visits of the same level of care, the most recent visit was selected [17]. Additional checks for data quality and random subsample review were performed for accuracy and consistency [17].

This study included patients who were 65 years of age or older at the time of ED presentation and followed them until completion of their hospital course. We excluded patients with incomplete REDCap records or missing length of stay fields. In total, 531 unique patients aged 65 and above with a positive result on PCR assay for SARS-CoV-2 who first presented to the ED or were hospitalized at NYP/CUIMC between March 6 and April 7, 2020 were included (Fig. 1).

\section{Statistical analysis}

We first characterized the prevalence of presenting signs and symptoms, excluding those with $<5 \%$ prevalence. Then, to examine the association between dementia status and in-hospital mortality, we created an unadjusted Kaplan-Meier curve and four Cox proportional hazards models: an unadjusted analysis, an analysis adjusted for age and sex, and two multivariate models adjusted for comorbidities, with/without code status as an additional covariate. The outcome was in-hospital death. Discharged patients were 
censored from the analysis at the time of discharge for the primary Cox analyses but were included in a sensitivity analysis of the final model with code status and in the Kaplan-Meier curve until the time of maximum length of stay (which was approximately 100 days) to account for potential bias.

Comorbidities adjusted for were based on those established by the CDC as risk factors for severe COVID-19 illness: active cancer, chronic kidney disease/end-stage renal disease (CKD/ESRD), chronic obstructive pulmonary disease, immunosuppressed state (defined as having had solid/liquid transplant, HIV, or being defined as "immunosuppressed" in the REDCap database), obesity (defined as BMI greater or equal to 30), coronary artery disease, heart failure, and diabetes (both types I and II) [19]. The total number of patients included in our two multivariate Cox models with/without code status was 482 patients, as obesity was adjusted for in these models, and 49 patients did not have data for BMI. No imputation was performed in this study. Cause-specific hazard ratios (HRs) from the Cox multivariate regression analyses were visualized with forest plots and reported with $95 \%$ confidence intervals and $p$-values for each covariate.

We used $\mathrm{R}$ version 4.0.2 for our statistical analysis [20-26]. Categorical variables were reported as proportions (\%) while continuous variables were reported as medians [interquartile range (IQR)]. For categorical variables, the $\chi^{2}$ test with Yates' continuity correction was used. Fisher's exact test was used for categorical variables that had a total prevalence of less than 5\%. Mann-Whitney U test was used for continuous variables. Statistically significant was considered $p<0.05$ in this study.

\section{RESULTS}

\section{Baseline characteristics}

We identified 531 patients at least 65 years of age at presentation who were hospitalized with COVID-19. There were 116 patients with previously diagnosed dementia, and 415 patients with no known history of dementia. Patients with previously diagnosed dementia were older (median 84.00, IQR [78.00-89.00] versus 73.00, IQR [69.00-80.00], $p<0.001$ ), more often female ( $56.9 \%$ versus $45.1 \%, p=0.03$ ), and had a higher prevalence of DNR/DNI orders at discharge or death when compared to patients with no known history of dementia $(60.3 \%$ versus $35.2 \%, p<0.001$; Table 1). There were no significant differences in race/ethnicity, smoking status, or comorbidities, except that more patients with previously diagnosed dementia had a prior cerebrovascular accident $(28.4 \%$ versus $10.8 \%, p<0.001)$ and none had a previous transplant (versus $4.1 \%$ for patients with no known history of dementia, $p=0.03$ ).

\section{Signs and symptoms}

Presenting signs and symptoms for COVID-19 differed among patients with and without dementia (Fig. 2). Patients with previously diagnosed dementia more often presented with delirium (36.2\% versus $11.6 \%, p<0.001$ ), and post-hoc multiple logistic regression found this remained significant after adjusting for age and sex (Supplementary Figure 1). These patients also less often manifested or communicated dyspnea $(45.7 \%$ versus $65.8 \%, p<0.001)$, diarrhea $(8.6 \%$ versus $21.9 \%, p=0.002)$, myalgias (8.6\% versus $21.0 \%, p=0.004)$, chills $(4.3 \%$ versus $19.3 \%, p<0.001)$, nausea/vomiting $(6.9 \%$ versus $16.9 \%, p=0.01)$, and headache $(0.9 \%$ versus $7.2 \%$, $p=0.02)$.

\section{Outcomes and in-hospital complications}

Patients with previously diagnosed dementia had higher in-hospital mortality in this cohort than patients with no known history of dementia $(50.0 \%$ versus $35.4 \%, p=0.006$; Table 2). They also had a shorter length of stay in days (median 4.60, IQR [2.21-7.55] versus 6.96, IQR [2.98-13.70], $p<0.001)$ and more often died earlier in their hospital course (Fig. 3A). A post-hoc subgroup analysis of all patients aged 78 or above (the lowest IQR boundary found for age in the dementia cohort) found that these differences in mortality and earlier hospital death did not remain significant once accounting for age as a confounder (Fig. 3B). There was no increase of inhospital complications for patients with previously diagnosed dementia; instead, patients with no known history of dementia had a higher incidence of septic shock $(23.6 \%$ versus $12.1 \%, p=0.01)$ and new onset arrhythmia (12.3\% versus 5.2\%, $p=0.04$; Table 2).

\section{Cox multivariate regression analysis}

In our multivariate Cox model without code status (Table 3, Model 3), the HR for dementia was 1.27 (95\% CI: 0.87-1.85). Covariates that showed statistically significant increased risk of in-hospital mortality in this model were age and CKD/ESRD, 
Table 1

Baseline Characteristics

\begin{tabular}{|c|c|c|c|c|c|}
\hline & $\begin{array}{c}\text { Total Patients } \\
\text { Age }=>65 \\
(n=531)\end{array}$ & $\begin{array}{c}\text { With Previously } \\
\text { Diagnosed Dementia } \\
(n=116)\end{array}$ & $\begin{array}{c}\text { With No Known } \\
\text { History of Dementia } \\
\quad(n=415)\end{array}$ & $p$ & Test $^{\mathrm{a}}$ \\
\hline Age (median [IQR]) & $76.00[70.00,83.00]$ & $84.00[78.00,89.00]$ & $73.00[69.00,80.00]$ & $<0.001$ & nonnorm \\
\hline BMI $(\text { median }[\mathrm{IQR}])^{\mathrm{b}}$ & $27.00[24.01,31.01]$ & $24.92[22.12,29.10]$ & $27.66[24.73,31.79]$ & $<0.001$ & nonnorm \\
\hline \multicolumn{6}{|l|}{ Sex $(\%)$} \\
\hline Male & $278(52.4)$ & $50(43.1)$ & $228(54.9)$ & 0.03 & \\
\hline Female & $253(47.6)$ & $66(56.9)$ & $187(45.1)$ & 0.03 & \\
\hline \multicolumn{6}{|l|}{ Race/Ethnicity $(\%)^{\mathrm{c}}$} \\
\hline White & $50(9.4)$ & $5(4.3)$ & $45(10.8)$ & 0.05 & \\
\hline Black & $56(10.5)$ & $12(10.3)$ & $44(10.6)$ & $>0.99$ & \\
\hline Asian & $4(0.8)$ & $0(0.0)$ & $4(1.0)$ & 0.58 & exact \\
\hline Other & $11(2.1)$ & $1(0.9)$ & $10(2.4)$ & 0.47 & exact \\
\hline Declined or Unknown $^{\mathrm{d}}$ & $128(24.1)$ & $31(26.7)$ & $97(23.4)$ & 0.53 & \\
\hline Hispanic & $282(53.1)$ & $67(57.8)$ & $215(51.8)$ & 0.30 & \\
\hline \multicolumn{6}{|l|}{ Code Status $(\%)^{\mathrm{e}}$} \\
\hline Full/Assumed Full & $315(59.3)$ & $46(39.7)$ & $269(64.8)$ & $<0.001$ & \\
\hline DNR/DNI & $216(40.7)$ & $70(60.3)$ & $146(35.2)$ & $<0.001$ & \\
\hline Prior Hospitalization & $46(21.3)$ & $28(40.0)$ & $18(12.3)$ & $<0.001$ & \\
\hline Prior to Admission ${ }^{\mathrm{f}}$ & $80(37.2)$ & $34(49.3)$ & $46(31.5)$ & 0.02 & \\
\hline Consistent DNR/DNI & $111(51.4)$ & $44(62.9)$ & $67(45.9)$ & 0.03 & \\
\hline Smoking Status (\%) & & & & 0.71 & \\
\hline No & $383(72.1)$ & $84(72.4)$ & $299(72.0)$ & & \\
\hline Active & $17(3.2)$ & $5(4.3)$ & $12(2.9)$ & & \\
\hline Former & $131(24.7)$ & $27(23.3)$ & $104(25.1)$ & & \\
\hline \multicolumn{6}{|l|}{ Comorbidities (\%) } \\
\hline HTN & $442(83.2)$ & $91(78.4)$ & $351(84.6)$ & 0.16 & \\
\hline DMI/DMII & $257(48.4)$ & $56(48.3)$ & $201(48.4)$ & $>0.99$ & \\
\hline HLD & $252(47.5)$ & 48 (41.4) & $204(49.2)$ & 0.17 & \\
\hline $\mathrm{CAD}$ & $111(20.9)$ & $30(25.9)$ & $81(19.5)$ & 0.18 & \\
\hline CKD/ESRD & $103(19.4)$ & $26(22.4)$ & 77 (18.6) & 0.43 & \\
\hline $\mathrm{HF}$ & $86(16.2)$ & $20(17.2)$ & $66(15.9)$ & 0.84 & \\
\hline CVA & $78(14.7)$ & $33(28.4)$ & $45(10.8)$ & $<0.001$ & \\
\hline COPD & $67(12.6)$ & $13(11.2)$ & $54(13.0)$ & 0.72 & \\
\hline Asthma & $47(8.9)$ & $6(5.2)$ & $41(9.9)$ & 0.16 & \\
\hline Active Cancer & $41(7.7)$ & $5(4.3)$ & $36(8.7)$ & 0.17 & \\
\hline Transplant & $17(3.2)$ & $0(0.0)$ & $17(4.1)$ & 0.03 & exact \\
\hline Cirrhosis & $10(1.9)$ & $1(0.9)$ & $9(2.2)$ & 0.70 & exact \\
\hline ILD & $8(1.5)$ & $1(0.9)$ & $7(1.7)$ & $>0.99$ & exact \\
\hline HIV & $6(1.1)$ & $2(1.7)$ & $4(1.0)$ & 0.62 & exact \\
\hline
\end{tabular}

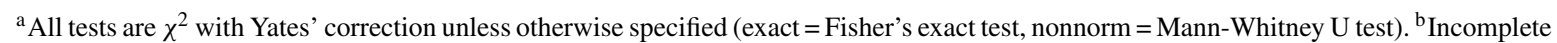
BMI reporting; $n=482,107$, and 375 . ' Each race category excludes patients of Hispanic, declined, unknown, or multi-racial ethnicity. ${ }^{\mathrm{d}}$ Includes declined race/ethnicity, unknown ethnicity, or multi-racial ethnicity. ${ }^{\mathrm{e}}$ Final code status at discharge or death. ${ }^{\mathrm{f}}$ One record missing date/time for DNR/DNI order; denominators are $n=215,69$, and 146 Sex, race/ethnicity, code status, smoking status, and comorbidities are reported for the total cohort in total prevalence (\%) format. Age and BMI are reported with medians and interquartile ranges. Proportion percentages for Prior Hospitalization, Prior to Admission, and Consistent DNR/DNI use the total prevalence of DNR/DNI orders at discharge or death as the denominator. Comorbidities are ordered from highest to lowest total prevalence. IQR, interquartile range; nonnorm, Mann-Whitney U test; exact, Fisher's exact test; DNR/DNI, Do-Not-Resuscitate/Do-Not-Intubate; HTN, hypertension; DMI/DMII, diabetes mellitus types I/II; HLD, hyperlipidemia; CAD, coronary artery disease; CKD/ESRD, chronic kidney disease/end-stage renal disease; HF, heart failure; CVA, cerebrovascular accident; COPD, chronic obstructive pulmonary disease; ILD, interstitial lung disease; HIV, human immunodeficiency virus.

while female sex conferred lower risk. After adding consistent DNR/DNI status to the model (Table 3, Model 4), dementia showed a similar HR to the model without code status $(\mathrm{HR}=1.29,95 \% \mathrm{CI}$ : $0.88-1.88$ ). Covariates which remained statistically significant in the multivariate Cox model with code status were age and female sex, but not CKD/ESRD.
Diabetes became significantly associated with increased in-hospital mortality, and the HR for consistent DNR/DNI status was 2.16 (95\% CI: 1.52-3.08). The significant covariates in the final multivariate Cox model with code status remained significant in the sensitivity analysis. All covariates are shown for the multivariate Cox models with and without code 


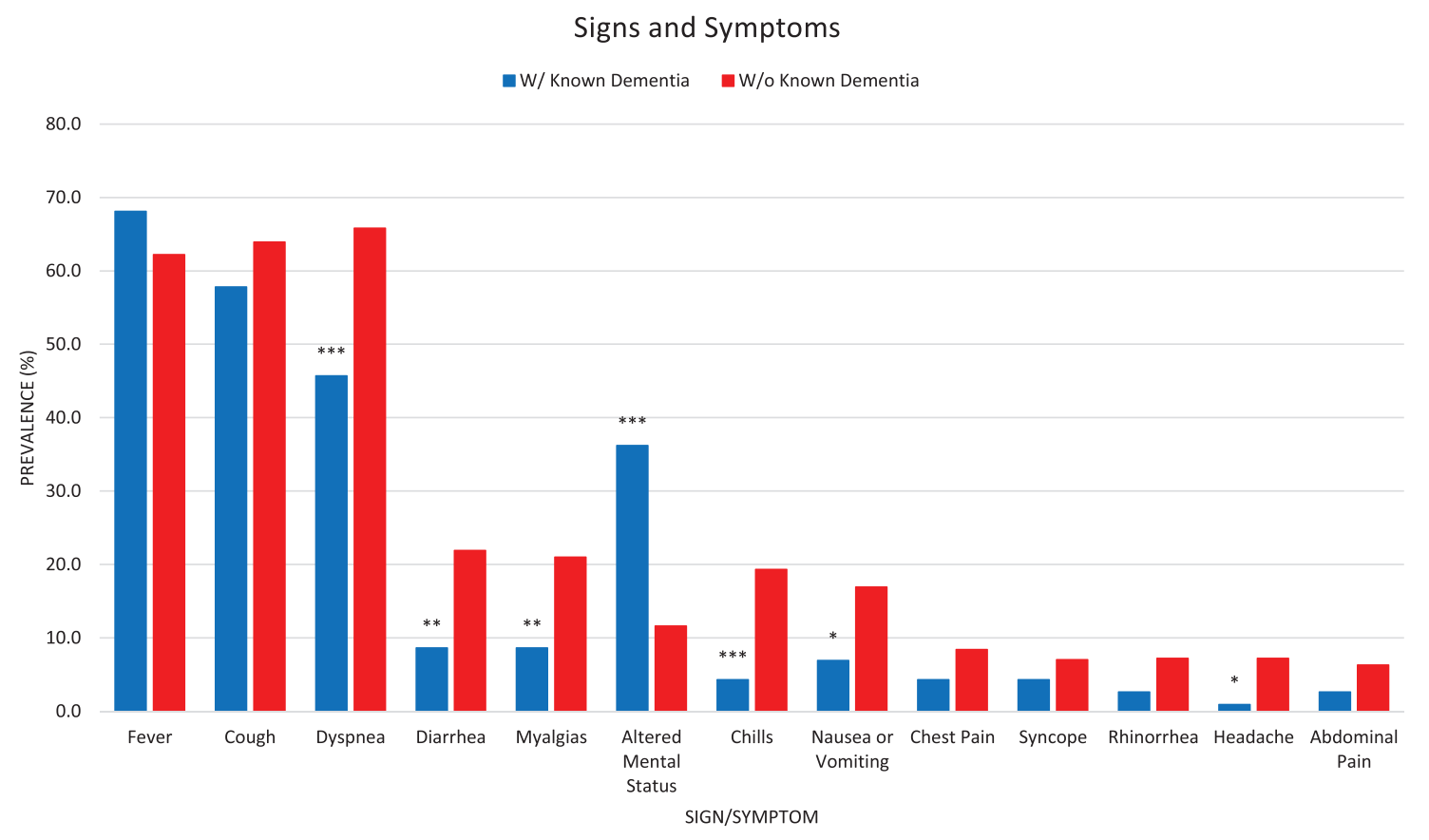

Fig. 2. Unadjusted Bar Graph for Signs and Symptoms. Signs and symptoms with greater than 5\% total prevalence are presented in a bar graph from highest to lowest prevalence in the total cohort. Patients with previously diagnosed dementia were more likely to present with delirium (altered mental status) and less likely to present with dyspnea, diarrhea, myalgias, chills, nausea/vomiting, and headache. Asterisks indicate statistical significance: ${ }^{* * *} p<0.001 ;{ }^{* *} 0.001<p<0.01 ;{ }^{*} 0.01<p<0.05$.

status in forest plots (Supplementary Figures 2 and $3 \mathrm{~A}$ and $3 \mathrm{~B})$.

\section{DISCUSSION}

In this cohort of consecutive patients who presented to the ED or were hospitalized with COVID19 , patients with previously diagnosed dementia had higher mortality and died earlier in their hospital course despite similar comorbidities and in-hospital complications, yet in multivariate analyses, dementia was not an independent risk factor for death. These patients manifested or communicated different signs and symptoms, with delirium being significantly more likely, while dyspnea, diarrhea, myalgias, chills, nausea/vomiting, and headache were less likely. These findings corroborate other studies that have found an increased prevalence of delirium in COVID19 patients with previously diagnosed dementia [6, 14-16], yet this study identifies for the first time the relative frequency of other more common COVID-19 signs and symptoms in persons with dementia hospitalized for COVID-19 and adjusts for age and sex in post-hoc analyses.

Improved recognition of the unique signs and symptoms of COVID-19 in patients with previously diagnosed dementia has potentially important public health implications. Identifying a dementia-specific COVID-19 profile could help family members, caregivers, and healthcare workers recognize COVID-19 illness more promptly, which could meaningfully impact health seeking behaviors and outcomes. New confusion is now a CDC emergency warning sign in COVID-19 [27], and findings from this cohort suggest a frequency of approximately one third of dementia patients presenting with delirium, here $36.2 \%$. A recent US study identified delirium as the presenting sign in seven EDs across the Northeast and South in $38 \%$ of patients with dementia and COVID19 [14], and an earlier study from the initial Italian COVID-19 wave identified delirium as the initial sign in $36.8 \%$ of dementia patients, with these patients having higher mortality [15]. In another Italian cohort, COVID-19 associated delirium is associated with increased mortality but was not shown to be an independent risk factor [16].

Since patients with dementia manifested or communicated vastly different signs and symptoms of COVID-19 in this cohort, delayed recognition of COVID-19 illness could potentially lead to these patients presenting to the ED in a more advanced stage of their illness. This is corroborated by the 
Table 2

Outcomes

\begin{tabular}{|c|c|c|c|c|c|}
\hline & $\begin{array}{c}\text { Total Patients } \\
\text { Age }=>65 \\
(n=531)\end{array}$ & $\begin{array}{c}\text { With Previously } \\
\text { Diagnosed Dementia } \\
(n=116)\end{array}$ & $\begin{array}{c}\text { With No Known } \\
\text { History of Dementia } \\
\quad(n=415)\end{array}$ & $p$ & Test $^{\mathrm{a}}$ \\
\hline \multicolumn{6}{|l|}{ Status (\%) } \\
\hline Discharged & $326(61.4)$ & $58(50.0)$ & $268(64.6)$ & 0.006 & \\
\hline Died in Hospital & $205(38.6)$ & $58(50.0)$ & $147(35.4)$ & 0.006 & \\
\hline Intubated (at least once) & $135(25.4)$ & $19(16.4)$ & $116(28.0)$ & 0.02 & \\
\hline Extubated (at least once) ${ }^{\mathrm{b}}$ & $58(43.6)$ & $6(31.6)$ & $52(45.6)$ & 0.37 & \\
\hline Length of Stay $(\text { median }[\mathrm{IQR}])^{\mathrm{c}}$ & $6.05[2.75,11.86]$ & $4.60[2.21,7.55]$ & $6.96[2.98,13.70]$ & $<0.001$ & nonnorm \\
\hline \multicolumn{6}{|l|}{ Highest Level of Care (\%) } \\
\hline ED & $54(10.2)$ & $11(9.5)$ & $43(10.4)$ & 0.92 & \\
\hline Died in ED & $25(46.3)$ & $6(54.5)$ & $19(44.2)$ & 0.78 & \\
\hline Inpatient (not ICU) & $347(65.3)$ & $89(76.7)$ & $258(62.2)$ & 0.005 & \\
\hline Died Inpatient & $93(26.8)$ & $39(43.8)$ & $54(20.9)$ & $<0.001$ & \\
\hline ICU & $130(24.5)$ & $16(13.8)$ & $114(27.5)$ & 0.004 & \\
\hline Died in ICU & $87(66.9)$ & $13(81.2)$ & $74(64.9)$ & 0.31 & \\
\hline \multicolumn{6}{|l|}{ Complications in Hospital (\%) } \\
\hline ARDS & $216(40.7)$ & $50(43.1)$ & $166(40.0)$ & 0.62 & \\
\hline AKI & $190(35.8)$ & $34(29.3)$ & $156(37.6)$ & 0.13 & \\
\hline Septic Shock & $112(21.1)$ & $14(12.1)$ & $98(23.6)$ & 0.01 & \\
\hline Arrhythmia (new onset) & $57(10.7)$ & $6(5.2)$ & $51(12.3)$ & 0.04 & \\
\hline Ventilator-Associated Pneumonia & $31(5.8)$ & $3(2.6)$ & $28(6.7)$ & 0.14 & \\
\hline HF (new onset) & $12(2.3)$ & $1(0.9)$ & $11(2.7)$ & 0.48 & exact \\
\hline MI & $8(1.5)$ & $1(0.9)$ & $7(1.7)$ & $>0.99$ & exact \\
\hline
\end{tabular}

earlier in-hospital mortality seen in our KaplanMeier curve (Fig. 2). Mortality at the end of the study period for patients with previously diagnosed dementia was also significantly higher $(50.0 \%$ versus $35.4 \%, p=0.006$ ). COVID-19 associated mortality in dementia has varied in previously published cohorts including 62.2\% (Italy), 43.4\% (Spain), and 39.8\% (separate New York City cohort) [6-8], with some large, multicenter studies finding dementia to be an independent risk factor for in-hospital mortality in multivariate analyses $[3,4,11]$.

Our cohort demonstrated similar rates of comorbidities and in-hospital complications between patients with and without previously diagnosed dementia (Tables 1 and 2). In fully adjusted models, dementia was not an independent risk factor for inhospital mortality, which differs from the results of other multicenter studies $[3,4,11]$. This difference could be explained by the limited sample size and generalizability of our single-center study; however, age, male sex, CKD/ESRD, and diabetes were independently associated with in-hospital mortality in our multivariate Cox models (Table 3, Models 3 and 4), which is consistent with what has been reported in the literature $[3,4,13]$. Given that patients with previously diagnosed dementia were older (Table 1), age may have been the stronger determinant of increased in-hospital mortality in our study. To this point, age was independently associated with mortality in all models. Further, in a post hoc analysis of those aged 78 years and older $(n=231,43.5 \%$ of the overall cohort), history of dementia was not independently associated with mortality. Although this older subgroup is smaller, and thus more limited power, mortality rates between the two groups were not significant $(53.3 \%$ mortality with dementia versus $46.8 \%$ without dementia, $p=0.41$, Fig. 3B).

Since code status can be an independent predictor of mortality $[28,29]$, we investigated whether it would dramatically impact or confound the outcomes for patients with dementia by including it as a covariate in our final model (Table 3, Model 4; Supplementary Figure 3A, B). DNR/DNI code status is as high as $49.8 \%$ in dementia based on a 5-state nursing home cohort [30]. Similarly, we identified DNR/DNI at discharge or death occurred 


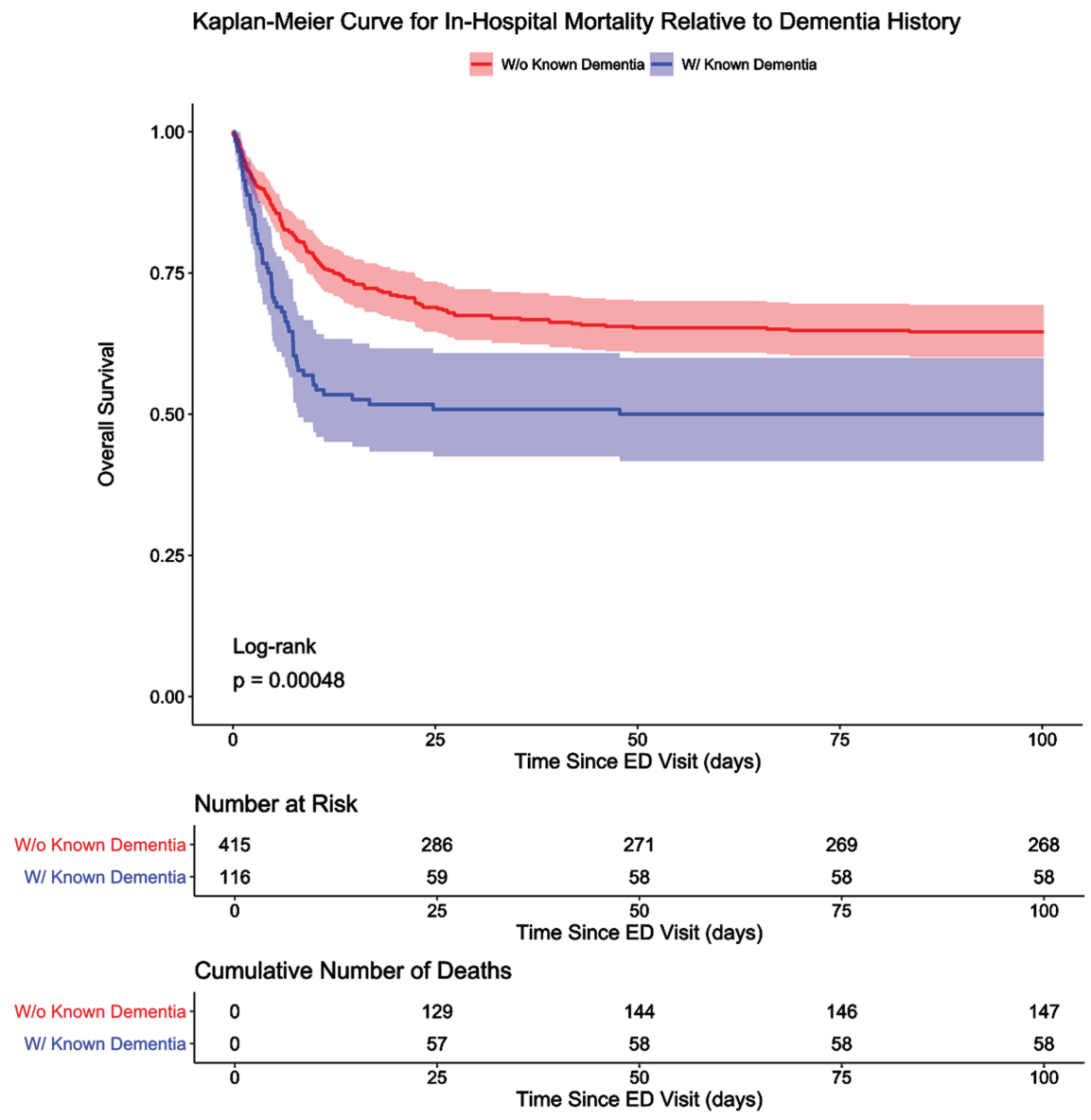

Fig. 3A. Kaplan-Meier Curve for In-Hospital Mortality Relative to Dementia History. Overall survival for patients with dementia and those with no known history of dementia are presented in an unadjusted Kaplan-Meier curve. Shading indicates $95 \%$ confidence intervals. Discharged alive patients were considered event-free and only censored at the maximum length of stay value in this study, which was approximately 100 days. Patients with dementia died earlier in their hospital course and had significantly lower overall survival than patients with no known history of dementia.

more frequently in patients with previously diagnosed dementia $(60.3 \%$ versus $35.2 \%, p<0.001)$. Our study uniquely accounts for consistent DNR/DNI status in COVID-19 patients with previously diagnosed dementia, and its inclusion in the fully adjusted model did not substantially attenuate most other covariates, except for CKD/ESRD. Those with consistent DNR/DNI status had a more than double the risk of in-hospital mortality, and dementia remained a non-significant risk factor for mortality in this fully adjusted model.

Taken together, our results suggest that the increased in-hospital mortality in the dementia cohort was associated with dementia patients being older rather than dementia itself, which is a reassuring finding. Yet, dementia patients still manifested or communicated vastly different signs and symptoms of COVID-19, which could hypothetically lead to decreased recognition and delayed treatment. Hence, improved recognition of COVID-19 and strategies to prevent exposure to SARS-CoV-2 in this vulnerable population are needed, with careful consideration for potential mental health problems and psychosocial support when implementing these strategies [1, 31, 32].

\section{Limitations}

There are several important limitations in this study. First, dementia diagnoses are well recognized as being historically underreported in medical records [33], and the dementia indicator in this dataset could instead be a subset mostly comprised of those with more apparent, advanced stages of dementia. However, the prevalence of dementia in this cohort is similar to well-established estimates 


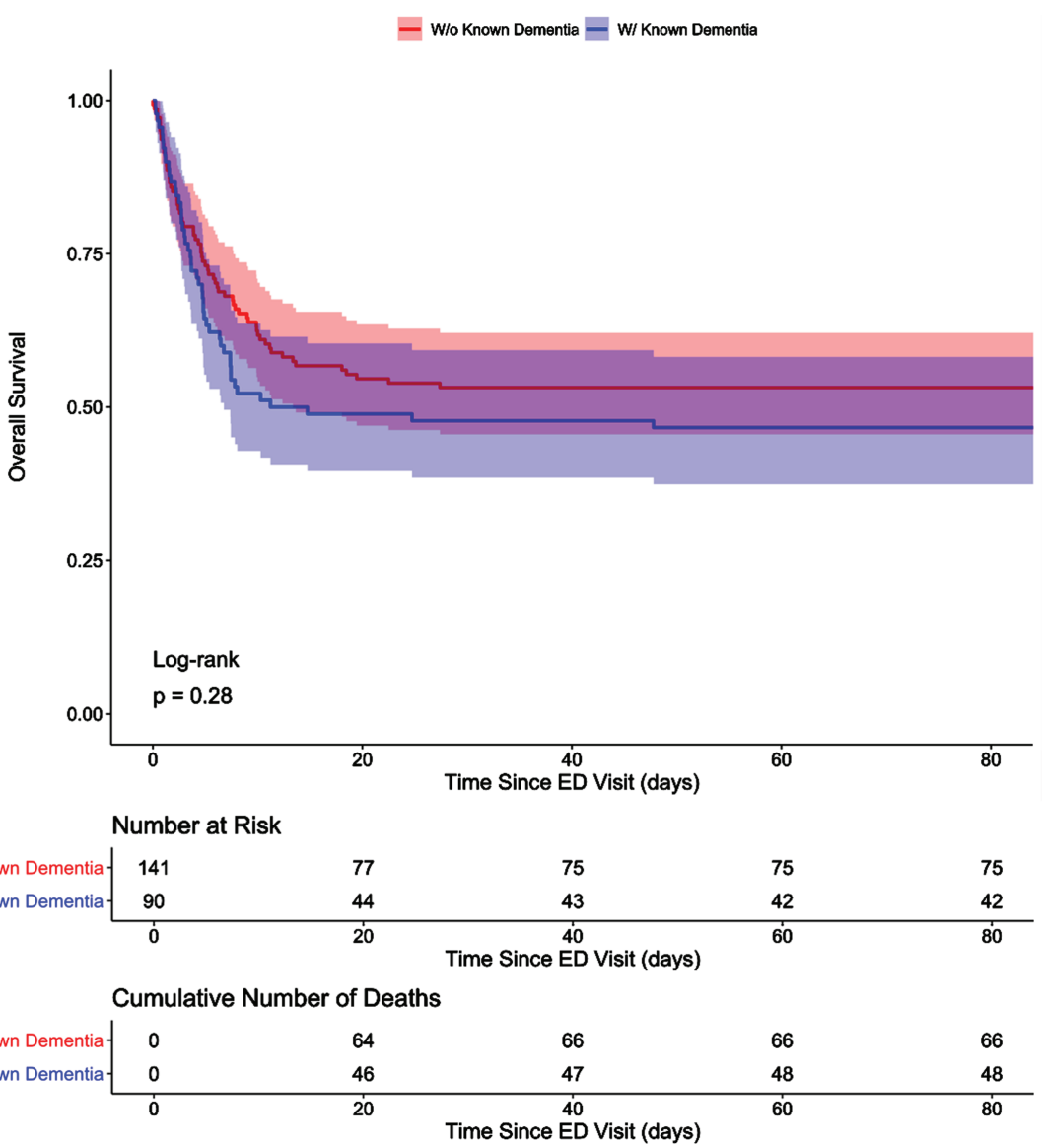

Fig. 3B. Kaplan-Meier Curve for In-Hospital Mortality Relative to Dementia History, Subgroup of All Patients Aged 78 or Above. Post-hoc subgroup analysis of overall survival for patients with dementia and those with no known history of dementia aged 78 or above are presented in a Kaplan-Meier curve. Shading indicates 95\% confidence intervals. Discharged alive patients were considered event-free and only censored at the maximum length of stay value in the subgroup of patients aged 78 or above, which was approximately 89 days. Earlier in-hospital death for patients with dementia was no longer significant in this analysis, and mortality at the end of the study period for both cohorts was also no longer statistically significant $(53.3 \%$ versus $46.8 \%, p=0.41)$.

Table 3

Cox Multivariate Regression Analysis

\begin{tabular}{|c|c|c|c|c|c|c|}
\hline & Age & Female & Diabetes & CKD/ESRD & Dementia & DNR/DNI \\
\hline Model $1^{\mathrm{a}}$ & - & - & - & - & $\begin{array}{c}2.20 \\
(1.60-3.00)\end{array}$ & - \\
\hline Model $2^{b}$ & $\begin{array}{c}1.08 \\
(1.06-1.10)\end{array}$ & $\begin{array}{c}0.69 \\
(0.52-0.93)\end{array}$ & - & - & $\begin{array}{c}1.30 \\
(0.92-1.83)\end{array}$ & - \\
\hline Model $3^{c}$ & $\begin{array}{c}1.08 \\
(1.06-1.10)\end{array}$ & $\begin{array}{c}0.69 \\
(0.50-0.94)\end{array}$ & $\begin{array}{c}1.33 \\
(0.98-1.80)\end{array}$ & $\begin{array}{c}1.52 \\
(1.05-2.20)\end{array}$ & $\begin{array}{c}1.27 \\
(0.87-1.85)\end{array}$ & - \\
\hline Model $4^{\mathrm{d}}$ & $\begin{array}{c}1.07 \\
(1.04-1.09)\end{array}$ & $\begin{array}{c}0.67 \\
(0.49-0.91)\end{array}$ & $\begin{array}{c}1.49 \\
(1.10-2.03)\end{array}$ & $\begin{array}{c}1.32 \\
(0.91-1.92)\end{array}$ & $\begin{array}{c}1.29 \\
(0.88-1.88)\end{array}$ & $\begin{array}{c}2.16 \\
(1.52-3.08)\end{array}$ \\
\hline
\end{tabular}

${ }^{\mathrm{a}}$ Model 1: Unadjusted analysis $(n=531) .{ }^{\mathrm{b}}$ Model 2: Adjusted for age and sex $(n=531) .{ }^{\mathrm{c}}$ Model 3: Model $2+$ CDC-defined comorbidities for severe COVID-19 illness $\left(n=482,49\right.$ missing BMI). ${ }^{\mathrm{d}}$ Model 4: Model $3+$ consistent DNR/DNI status $(n=482,49$ missing BMI) Hazard ratios with their $95 \%$ confidence intervals are reported for age, female sex, diabetes, chronic kidney disease/end-stage renal disease, dementia, and consistent Do-Not-Resuscitate/Do-Not-Intubate status in the four models created for this analysis. All bolded hazard ratios are significant at the $5 \%$ significance level. CKD/ESRD, chronic kidney disease/end-stage renal disease; DNR/DNI, Do-Not-Resuscitate/Do-NotIntubate. 
in the same community served by this hospital [34, 35]. The separation of symptoms in multiple domains relative to dementia lends further credence to an accurate diagnosis. Although the record review extracted meaningful clinical data about acute neuropsychiatric symptoms, a formal determination of toxic metabolic delirium due to COVID-19 cannot be definitively determined. Moreover, despite efforts to specifically identify new symptoms of delirium, it is possible that for some patients, the symptoms identified in data extraction are simply reflective of underlying dementia.

Given that the most common sign in dementia is memory loss, this may contribute to poor recognition and underreporting of COVID-19 symptoms; with the exception of fever and delirium, all other COVID-19 symptoms were less often evident in patients with dementia. Additionally, obtaining an accurate history from a patient with dementia could have been more difficult. This was especially pertinent in the context of a busy ED during a pandemic, where family members and caregivers may not have accompanied patients or were primarily engaged by phone or virtual means, and thus introduced challenges with the history-taking process. Nonetheless, these findings reflect the way in which care was delivered and recorded in the imperfect reality of the early days of what became the epicenter of the US COVID-19 epidemic, which increasingly correlates with care being delivered around the world in a worsening pandemic.

While dementia was not a statistically independent risk factor for in-hospital death, our study was limited in sample size and may have been under-powered to detect some outcomes. Additionally, there could be other residual confounders in our analyses. Race and ethnicity are well recognized as complex surrogates for social determinants of health; they could not be included in our models because of missing data for many in the cohort. Nonetheless, significant associations were identified in other established comorbidities for severe COVID-19 illness, namely CKD/ESRD and diabetes [19].

While code status was associated with in-hospital mortality in this cohort, illness severity at the time of a DNR/DNI order could not be accounted for in our model and could be an important residual confounder [36]. Following a diagnosis of a potentially severe illness like COVID-19, advanced directives are more likely to be discussed, and this becomes even more the case should the illness become severe or appear futile. With the premise that discussions of advanced directives are clinically indicated and ethically justified, it is likely that severe illness is driving increased in-hospital mortality rather than code status [36]. These code status discussions were especially challenging in early periods of COVID-19 care in NYC when hospital resources, particularly ventilators, were in short supply. Other centers may have faced similar challenges during the COVID-19 wave of winter 2021 as well as other contexts. Aside from controlling for code status in regression models, we considered alternative approaches such as inversion propensity weighting. However, such an approach presents pragmatic challenges including sample size which was too small to sufficiently enable appropriate balance across all covariates. Moreover, any additional modeling would not be able to further manage the challenging contextual determinants of code status that we have highlighted.

Finally, potential errors could have occurred during the manual abstraction of the EMR into the REDCap database, although quality control checks were performed to minimize these errors. All findings in this study were from a single urban medical center, which limited the sample size and generalizability. It is also recognized that collective understanding of how to effectively treat COVID-19 has evolved since this cohort was encountered early in the US pandemic.

\section{CONCLUSION}

In this study, COVID-19 patients with previously diagnosed dementia had higher in-hospital mortality, but dementia was not an independent risk factor for in-hospital death in fully adjusted models. These patients were significantly more likely to present with delirium and less likely to manifest or communicate dyspnea, diarrhea, myalgias, chills, nausea/vomiting, and headache than older adults without dementia, even after adjusting for age and sex. Special attention is needed to prevent SARS-CoV-2 infection in these patients, recognize COVID-19 illness if infected, and ensure that social support is maintained for these patients in the event of isolation or hospitalization.

\section{ACKNOWLEDGMENTS}

We would like to thank the frontline workers for their dedication and sacrifice to the lives of the many patients and families who were affected by the COVID-19 crisis. We are also grateful to the many patients who sought care at our medical center and provided the information in the EMR that was essential for this research study. Finally, we would 
like to thank the Weill Cornell Medicine COVID-19 Registry Team, which designed the REDCap instrument used in this study and assisted with training [17], and the medical students at the Columbia University Vagelos College of Physicians and Surgeons, who manually abstracted data from the EMR during the peak months of the pandemic and helped create the REDCap database that was used in this study. The names of the medical students are as follows: Michael G. Argenziano, Samuel L. Bruce, Cody L. Slater, Jonathan R. Tiao, Paul A. Asadourian, Fletcher M. Bell, Rebekah Boyd, Matthew F. Cohen, MacAlistair I. Colquhoun, Lucy A. Colville, Joseph H. de Jonge, Lyle B. Dershowitz, Shirin A. Dey, Katherine A. Eiseman, Zachary P. Girvin, Daniella T. Goni, Nicholas Herzik, Sarah Householder, Lara E. Karaaslan, Heather Lee, Evan Lieberman, Andrew Ling, Ree Lu, Arthur Y. Shou, Alexander C. Sisti, Zachary E. Snow, Colin.P Sperring, Yuqing Xiong, and Henry W. Zhou.

No specific funding or grants were received for the prior study of which this research was based on. This study and Mr. Harb were supported by grant T35 AG044303 from the National Institute on Aging. Dr. Noble receives support from P30 AG066462, R01 AG054536, and T35 AG044303.

Authors' disclosures available online (https:// www.j-alz.com/manuscript-disclosures/21-0050r1).

\section{SUPPLEMENTARY MATERIAL}

The supplementary material is available in the electronic version of this article: https://dx.doi.org/ 10.3233/JAD-210050.

\section{REFERENCES}

[1] Wang H, Li T, Barbarino P, Gauthier S, Brodaty H, Molinuevo JL, Xie H, Sun Y, Yu E, Tang Y, Weidner W, Yu $X$ (2020) Dementia care during COVID-19. Lancet 395, 1190-1191.

[2] Centers for Disease Control and Prevention (2021) Excess deaths associated with COVID-19. https://www.cdc.gov/ nchs/nvss/vsrr/covid19/excess_deaths.htm, Last updated January 9, 2021, Accessed on January 9, 2021.

[3] Harrison SL, Fazio-Eynullayeva E, Lane DA, Underhill P, Lip GYH (2020) Comorbidities associated with mortality in 31,461 adults with COVID-19 in the United States: A federated electronic medical record analysis. PLoS Med 17, e1003321.

[4] Atkins JL, Masoli JAH, Delgado J, Pilling LC, Kuo CL, Kuchel GA, Melzer D (2020) Preexisting comorbidities predicting COVID-19 and mortality in the UK Biobank Community Cohort. J Gerontol A Biol Sci Med Sci 75, 2224-2230.
[5] van Gerwen M, Alsen M, Little C, Barlow J, Genden E, Naymagon L, Tremblay D (2021) Risk factors and outcomes of COVID-19 in New York City; a retrospective cohort study. J Med Virol 93, 907-915.

[6] Bianchetti A, Rozzini R, Guerini F, Boffelli S, Ranieri P, Minelli G, Bianchetti L, Trabucchi M (2020) Clinical presentation of COVID19 in dementia patients. $J$ Nutr Health Aging 24, 560-562.

[7] Reyes-Bueno JA, Mena-Vázquez N, Ojea-Ortega T, Gonzalez-Sotomayor MM, Cabezudo-Garcia P, CianoPetersen NL, Pons-Pons G, Castro-Sánchez MV, SerranoCastro PJ (2020) Case fatality of COVID-19 in patients with neurodegenerative dementia. Neurologia 35, 639-645.

[8] Miyashita S, Yamada T, Mikami T, Miyashita H, Chopra N, Rizk D (2020) Impact of dementia on clinical outcomes in elderly patients with coronavirus 2019 (COVID-19): An experience in New York. Geriatr Gerontol Int 20, 732-734.

[9] Hwang JM, Kim JH, Park JS, Chang MC, Park D (2020) Neurological diseases as mortality predictive factors for patients with COVID-19: A retrospective cohort study. $\mathrm{Neu}$ rol Sci 41, 2317-2324.

[10] Covino M, De Matteis G, Santoro M, Sabia L, Simeoni B, Candelli M, Ojetti V, Franceschi F (2020) Clinical characteristics and prognostic factors in COVID-19 patients aged $\geq 80$ years. Geriatr Gerontol Int 20, 704-708.

[11] Esme M, Koca M, Dikmeer A, Balci C, Ata N, Dogu BB, Cankurtaran M, Yilmaz M, Celik O, Unal GG, Ulgu MM, Birinci S (2021) Older adults with coronavirus disease 2019; A nationwide study in Turkey. J Gerontol A Biol Sci Med Sci 76, e68-e75.

[12] Tsugawa A, Sakurai S, Inagawa Y, Hirose D, Kaneko Y, Ogawa Y, Serisawa S, Takenoshita N, Sakurai H, Kanetaka H, Hirao K, Shimizu S (2020) Awareness of the COVID-19 outbreak and resultant depressive tendencies in patients with severe Alzheimer's disease. J Alzheimers Dis 77, 539-541.

[13] Williamson EJ, Walker AJ, Bhaskaran K, Bacon S, Bates C, Morton CE, Curtis HJ, Mehrkar A, Evans D, Inglesby P, Cockburn J, McDonald HI, MacKenna B, Tomlinson L, Douglas IJ, Rentsch CT, Mathur R, Wong AYS, Grieve R, Harrison D, Forbes H, Schultze A, Croker R, Parry J, Hester F, Harper S, Perera R, Evans SJW, Smeeth L, Goldacre B (2020) Factors associated with COVID-19-related death using OpenSAFELY. Nature 584, 430-436.

[14] Kennedy M, Helfand BKI, Gou RY, Gartaganis SL, Webb M, Moccia JM, Bruursema SN, Dokic B, McCulloch B, Ring H, Margolin JD, Zhang E, Anderson R, Babine RL, Hshieh T, Wong AH, Taylor RA, Davenport K, Teresi B, Fong TG, Inouye SK (2020) Delirium in older patients with COVID-19 presenting to the emergency department. JAMA Netw Open 3, e2029540.

[15] Poloni TE, Carlos AF, Cairati M, Cutaia C, Medici V, Marelli E, Ferrari D, Galli A, Bognetti P, Davin A, Cirrincione A, Ceretti A, Cereda C, Ceroni M, Tronconi L, Vitali S, Guaita A (2020) Prevalence and prognostic value of delirium as the initial presentation of COVID-19 in the elderly with dementia: An Italian retrospective study. EClinicalMedicine 26, 100490.

[16] Ticinesi A, Cerundolo N, Parise A, Nouvenne A, Prati B, Guerra A, Lauretani F, Maggio M, Meschi T (2020) Delirium in COVID-19: Epidemiology and clinical correlations in a large group of patients admitted to an academic hospital. Aging Clin Exp Res 32, 2159-2166.

[17] Argenziano MG, Bruce SL, Slater CL, Tiao JR, Baldwin MR, Barr RG, Chang BP, Chau KH, Choi JJ, Gavin N, Goyal P, Mills AM, Patel AA, Romney MS, Safford MM, Schluger 
NW, Sengupta S, Sobieszczyk ME, Zucker JE, Asadourian PA, Bell FM, Boyd R, Cohen MF, Colquhoun MI, Colville LA, de Jonge JH, Dershowitz LB, Dey SA, Eiseman KA, Girvin ZP, Goni DT, Harb AA, Herzik N, Householder S, Karaaslan LE, Lee H, Lieberman E, Ling A, Lu R, Shou AY, Sisti AC, Snow ZE, Sperring CP, Xiong Y, Zhou HW, Natarajan K, Hripcsak G, Chen R (2020) Characterization and clinical course of 1000 patients with coronavirus disease 2019 in New York: Retrospective case series. BMJ 369, m1996.

[18] Harris PA, Taylor R, Thielke R, Payne J, Gonzalez N, Conde JG (2009) Research electronic data capture (REDCap)a metadata-driven methodology and workflow process for providing translational research informatics support. $J$ Biomed Inform 42, 377-381.

[19] Centers for Disease Control and Prevention (2020) Certain medical conditions and risk for severe COVID-19 illness. https://www.cdc.gov/coronavirus/2019-ncov/need-extraprecautions/people-with-medical-conditions.html, Last updated December 29, 2020, Accessed on January 9, 2021.

[20] R Development Core Team (2010) R: A language and environment for statistical computing. R Foundation for Statistical Computing.

[21] Therneau T (2020) A Package for Survival Analysis in R. R package version 3.2-7, https://CRAN.R-project.org/ package $=$ survival.

[22] Barnier J, Briatte F, Larmarange J (2020) questionr: Functions to make surveys processing easier. R package version 0.7.3. https://CRAN.R-project.org/package=questionr

[23] Kassambara A, Kosinski M, Biecek P (2020) survminer: Drawing survival curves using 'ggplot2'. R package version 0.4.8. https://CRAN.R-project.org/package=survminer

[24] Gordon M, Lumley T (2020) forestplot: Advanced forest plot using 'grid' graphics. R package version 1.10. https://CRAN.R-project.org/package $=$ forestplot

[25] Iannone R (2020) DiagrammeR: Graph/network visualization. $\mathrm{R}$ package version 1.0.6.1. https://CRAN.Rproject.org/package $=$ DiagrammeR

[26] Yoshida K, Bartel A (2020) tableone: Create 'Table 1' to describe baseline characteristics with or without propensity score weights. R package version 0.12.0. https://CRAN.Rproject.org/package=tableone
[27] Centers for Disease Control and Prevention (2020) Symptoms of coronavirus. https://www.cdc.gov/coronavirus/ 2019-ncov/symptoms-testing/symptoms.html, Last updated December 22, 2020, Accessed on January 9, 2021.

[28] Fuchs L, Anstey M, Feng M, Toledano R, Kogan S, Howell MD, Clardy P, Celi L, Talmor D, Novack V (2017) Quantifying the mortality impact of do-not-resuscitate orders in the ICU. Crit Care Med 45, 1019-1027.

[29] Shepardson LB, Youngner SJ, Speroff T, Rosenthal GE (1999) Increased risk of death in patients with do-notresuscitate orders. Med Care 37, 727-737.

[30] Tjia J, Dharmawardene M, Givens JL (2018) Advance directives among nursing home residents with mild, moderate, and advanced dementia. $J$ Palliat Med 21, 16-21.

[31] El Haj M, Altintas E, Chapelet G, Kapogiannis D, Gallouj K (2020) High depression and anxiety in people with Alzheimer's disease living in retirement homes during the covid-19 crisis. Psychiatry Res 291, 113294.

[32] Giebel C, Lord K, Cooper C, Shenton J, Cannon J, Pulford D, Shaw L, Gaughan A, Tetlow H, Butchard S, Limbert S, Callaghan S, Whittington R, Rogers C, Komuravelli A, Rajagopal M, Eley R, Watkins C, Downs M, Reilly S, Ward K, Corcoran R, Bennett K, Gabbay M (2021) A UK survey of COVID-19 related social support closures and their effects on older people, people with dementia, and carers. Int J Geriatr Psychiatry 36, 393-402.

[33] Zhu CW, Ornstein KA, Cosentino S, Gu Y, Andrews H, Stern Y (2019) Misidentification of dementia in Medicare claims and related costs. J Am Geriatr Soc 67, 269-276.

[34] Brickman AM, Schupf N, Manly JJ, Luchsinger JA, Andrews H, Tang MX, Reitz C, Small SA, Mayeux R, DeCarli C, Brown TR (2008) Brain morphology in older African Americans, Caribbean Hispanics, and whites from northern Manhattan. Arch Neurol 65, 1053-1061.

[35] Tang MX, Stern Y, Marder K, Bell K, Gurland B, Lantigua R, Andrews H, Feng L, Tycko B, Mayeux R (1998) The APOE-epsilon4 allele and the risk of Alzheimer disease among African Americans, whites, and Hispanics. JAMA 279, 751-755.

[36] Chen YY, Chen YS, Chu TS, Lin KH, Wu CC (2016) Further deliberating the relationship between do-not-resuscitate and the increased risk of death. Sci Rep 6, 23182. 